\title{
Analytical computation of completion time distributions of short-lived TCP connections is
}

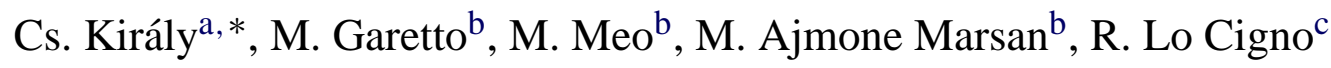 \\ ${ }^{a}$ Department of Telecommunications, Budapest University of Technology and Economics, Budapest, Hungary \\ b Dipartimento di Elettronica, Politecnico di Torino, Torino, Italy \\ ${ }^{\mathrm{c}}$ Dipartimento di Informatica e Telecomunicazioni, Università di Trento, Trento, Italy
}

Available online 11 September 2004

\begin{abstract}
A new technique for the analytical evaluation of distributions (and quantiles) of the completion time of shortlived TCP connections is presented and discussed. The proposed technique derives from known open multiclass queuing network (OMQN) models of the TCP protocol and computes a discrete approximation, with arbitrary accuracy, of the distribution of sojourn times of customers in the OMQN, which corresponds to the distribution of completion times of the modeled TCP connections. The proposed technique is computationally efficient, and its asymptotic complexity is independent of the network topology, of the number of concurrent flows, and of other network parameters.
\end{abstract}

(C) 2004 Elsevier B.V. All rights reserved.

Keywords: TCP; Queuing networks; Completion time distribution; Analytical models

\section{Introduction}

Three facts suggest that the most interesting metrics for Internet design and planning relate to the TCP connection completion time: (I) IP networks now support a wide range of services with variable quality of service (QoS) requirements; (II) over $90 \%$ of the packet traffic over the Internet is generated by

\footnotetext{
it A preliminary version of this paper was presented at the HET-NETs'03 Conference, Ilkley, West Yorkshire, UK, July 2003.

* Corresponding author.

E-mail addresses: cskiraly@ hit.bme.hu (Cs. Király); garetto@polito.it (M. Garetto); michela@polito.it (M. Meo); ajmone@polito.it (M. Ajmone Marsan); locigno@dit.unitn.it (R. Lo Cigno)
} 
applications using the TCP protocol; (III) end-users are mostly sensitive to the time needed to complete their data transfer, irrespective of how this is accomplished (e.g., a customer is not satisfied knowing that the packet drop rate in the network is very low, if his own transfer takes a long time to complete).

Not many techniques exist for the design of packet networks, and network operators still often rely on the approaches devised in the 1970s, that assume Poisson packet flows, exponential packet lengths, and independence. In addition, they consider the average packet delay as the only target of network design, which is not easily related to the end-user perceived performance.

For this reason, in recent years, the development of analytical models of TCP has become a hot research topic. Researchers examined the throughput achievable by long-lived connections [1,3,16], and, even more importantly, the completion time of short-lived connections $[4,13,17,18]$. Several papers proposed methods to obtain the average completion time of short-lived TCP connections (a concise overview of previous work is presented in the next section). However, the variability of QoS requirements for different types of services, hence different types of TCP connections, and the need to provide service level agreements (SLAs) to end users, require the computation of more sophisticated performance metrics, such as completion time distributions, and quantiles of the completion time for TCP connections that need to transfer a given number of segments.

In this paper, we propose an efficient analytical technique for the computation of completion time distributions based on open multiclass queuing network (OMQN) models of the TCP protocol [5-9].

An OMQN model is a stochastic representation of the finite state machine (FSM) description of the TCP transmitter behavior, and provides a precise model of the TCP dynamics. It requires as input the average packet loss probability, and the average round trip time for the TCP connections being considered. The OMQN model decouples the description of the protocol behavior from the description of the network behavior, so that the above two parameters can be obtained in several different ways: (i) they can be measured over an actual network or an experimental setup, (ii) they can be obtained from simulation experiments, (iii) they can be estimated with an analytical model of the underlying IP network; (iv) they can be set by the network designer to find planning requirements and operating points.

This flexibility allows OMQN models to be used in any networking scenario. For instance, in designing a mixed wired and wireless network, the design objectives can be divided between the wired and the wireless part. From an analytical perspective, decoupling the TCP model from the network model enables to tackle complex, heterogeneous scenarios that are inhibited by more structured approaches where the behavior of TCP and network are modeled jointly. See for instance the joint modeling of TCP with AQM routers in $[10,11,14]$.

The availability of a model to compute completion time distributions offers advantages, regardless of how the average packet loss probability and round trip time are obtained.

- It can be argued that, if the loss probability and round trip time are obtained by measurements, completion time distributions can also be measured (although this is significantly more complex). However, the advantage of the model described in this paper lies in allowing "what-if" analysis to assess the effectiveness of network upgrades.

- If the loss probability and round trip time are obtained by simulation, then the completion time distribution can also be estimated from the same simulation experiments. However, CPU times needed to obtain reliable estimates for distributions from simulations are exceedingly long. The technique we propose allows simulation to be used only to obtain parameters that are (relatively) easy to estimate accurately. Completion time distributions and their quantiles can then be computed analytically. The 
overall procedure is semi-analytic: some system parts are accurately modeled analytically (in our case TCP), while the parts that are too complex (non-linear, heterogeneous, etc.) are simulated.

- Finally, the completion time disribution computation can be embedded in a complete OMQN based analytical approach, operating as a post-processing procedure once the TCP and network models have been solved (see Section 3 and [5-7] for further details).

We emphasize here that the contribution of this work is neither the OMQN model of TCP (which was already presented in the literature [5-9]), nor an IP network model, rather a methodology to derive completion time quantiles and distributions. We deem this is a major step forward in analytical modeling for performance evaluation of end-to-end protocols, like TCP, and its application to multiservice networks. Given the close relationship between the OMQN and the FSM of a protocol, the extension of the presented technique to protocols different from TCP is straightforward.

The rest of this paper is organized as follows. Section 2 provides a concise overview of previous work on the estimation of short-lived TCP connection completion times. Section 3 briefly describes the open multiclass queuing network model of TCP. Section 4 introduces our technique for the computation of the completion time distribution of short-lived TCP connections. Section 5 presents numerical results both for the validation of the analytical approach and for the demonstration of the flexibility and the power of the proposed methodology. Finally, Section 6 contains our closing remarks.

\section{Previous work}

A vast literature exists on analytical models of TCP, thus we cannot provide here a comprehensive overview of previous contributions; rather, we mention some of the studies that address the estimation of the completion time of short-lived TCP transfers, particularly those that adopt techniques similar to the one used in this paper.

A large number of papers tackled the estimation of the steady-state throughput of infinite (or greedy) TCP flows (those that are today often called elephants), progressively introducing more and more refinements $[1,12,16]$. However, throughput alone is insufficient to characterize the behavior of some classes of end-user applications, and it is surely inappropriate for the characterization of the QoS offered to shortlived TCP flows (those that are today often called mice), whose performance is dominated by startup effects, such as connection establishment and initial slow start.

In [4], the authors extend the steady-state model of [16] to describe the behavior of finite TCP flows. In particular, they account for the connection establishment phase (whose duration distribution is estimated), and provide an approximate analysis of the first slow start phase, assuming correlated losses. Then, in order to obtain the time necessary to transfer any data remaining after the initial slow start phase, they resort to the steady-state throughput formula of [16]. Combining results, they obtain closed-form expressions for the average completion time of finite TCP flows, given as input parameters the TCP connection transfer size in bytes or segments, the TCP connection round trip time and packet loss probability.

The same approach was revisited and significantly extended in [17,18], using a more accurate model to describe the evolution of the congestion window during the initial slow start phase. In particular, the authors provide a very detailed analysis of all cases that can occur up to the first loss event and the corresponding recovery phase. In the case of multiple losses, they compute the conditional mean transfer time for a given number of loss events, assuming that losses are uniformly spaced, and that their 
number is binomially distributed. Both cases of independent and correlated losses are considered, as well as the behavior of different TCP versions [18]. A few additional assumptions and some empirical approximations are necessary to obtain expressions for the average completion time of arbitrarily long flows.

In [13], the authors use a different approach to derive the average completion time of short-lived TCP flows. Instead of dividing the transfer into different phases, they employ recursive equations that allow all the possible error patterns to be considered. In the case of i.i.d. losses, their results are very accurate, and their analysis is tractable up to about 10-packets TCP connections; longer connections quickly become intractable due to the exponential growth in the number of error patterns.

TCP connections completion time distributions were studied by simulation in [15]. There, the authors consider the impact of different models of loss correlation, and the effect of lost acknowledgments, suggesting that simplified Markov models could yield good approximations for the entire distribution as well as for the expectation.

In [2], Critical Path Analysis was applied to analyze packet traces obtained by tcpdump at the endpoints of HTTP connections, with the purpose of understanding what fraction of the total transfer latency is due to file size, packet propagation time, network variation (e.g., queueing delays at routers), packet losses or server load. The authors also investigated the causes of variability in transfer durations, showing that timeouts are the overwhelming contributor for small and medium sized files.

\section{Open multiclass queuing network model of TCP}

The behavior of TCP, like that of any protocol, can be specified through a finite state machine that describes the evolution of the protocol from one state to the next. Transitions between states are triggered by events (timers expiration, ACK reception, etc.). Actually, TCP is described through two FSMs, one for the transmitter and one for the receiver, but the protocol performance is driven essentially by the transmitter behavior, provided that the receiver buffer is not a bottleneck.

In [5-8], we proposed the use of open multiclass queuing networks to develop detailed models that can accurately estimate the performance of a number of competing TCP connections. The OMQN model $\mathcal{Q}=\left\{q_{i}\right\}$ comprises individual queues $q_{i}$ that describe the possible states of the FSM. Customers at a queue represent TCP connections in a given state. Queue service times $L\left(q_{i}\right)$ describe the permanence of connections in the corresponding state, and are associated with generally distributed random variables. ${ }^{1}$ Since there are no limits to the number of TCP connections in any given state (the connection state is a local variable), queues have infinite servers. The transition probability from $q_{i}$ to $q_{j}$ is defined as $T\left(q_{i}, q_{j}\right)$, and its value is derived from the protocol parameters and the underlying IP network characteristics. Since TCP connections are fairly independent from one another (neglecting synchronization phenomena and application-induced correlations), the arrival process in $q_{i}, \forall i$ is assumed to be Poisson. Hence, every queue in $\mathcal{Q}$ is an $M / G / \infty$ (or $M / D / \infty)$ queue.

The protocol behavior is completely described by the set $\left\{\mathcal{Q} ; L\left(q_{i}\right) ; T\left(q_{i}, q_{j}\right)\right\}, \forall i$, $j$; however, an important piece of information is still missing for the description of a whole TCP transfer: the backlog

\footnotetext{
${ }^{1}$ The queue service times $L\left(q_{i}\right)$ mainly depend on the TCP connection round trip time; hence, the accurate characterization of service times would require the knowledge of the round trip time distribution. Such distributions are rarely available, but it is possible to use constant service times calculated from the average round trip time.
} 
of the connection, i.e., the residual number of segments to be transmitted to complete the transfer. This information is associated with customers (i.e., TCP connections) through a class $c \in[0, N]$ where $N$ is the number of segments to be transferred when the connection opens. A new customer (TCP connection) enters the system in queue $\mathrm{FE}_{1}{ }^{2}$ (identical for all connections) describing the initial state for all TCP connections, with class $c=N$. The class $c$ of a customer is decremented whenever the customer visits a queue which represents the successful transmission of a segment; when a customer reaches $c=0$, it enters a 'closing queue' $q_{M}$, and then leaves the system. This latter queue can for instance represent the half-close procedure of TCP. Any connection in the system is completely described by the state $\mathbf{s}=(q, c)$.

Since queues have an infinite number of servers, the steady-state solution of the OMQN model reduces to solving the flow balance equations, hence it is computationally very light. The model can be solved either by having as inputs the network parameters, basically the packet loss probability $P_{L}$ and the average round trip time $\overline{\mathrm{RTT}}$, or by jointly solving a network model with a fixed point approximation (FPA): given $P_{L}$ and $\overline{\mathrm{RTT}}$, the OMQN provides the load to the network, which in turn returns new values of $P_{L}$ and $\overline{\mathrm{RTT}}$. The convergence of the FPA is proven in [9].

For further details on OMQN modeling, results, and FPA iterative solution, we refer the reader to the cited papers.

\subsection{Customer's behavior in the OMQN model}

In $[7,8]$, average TCP connection completion times were computed using Little's result, starting from the average number of customers (TCP connections) in the OMQN. However, the OMQN model contains much more information that can be used, as explained in Section 4, to compute the distribution of the time necessary to complete the transfer of a file with given length through a TCP connection. First of all, notice that all customers (TCP connections) enter the OMQN in the same queue $\mathrm{FE}_{1}$, and, after visiting an arbitrary number of queues and classes, exit the OMQN from the same state $\mathbf{s}=\left(q_{M}, 0\right)$.

Fig. 1 depicts a small portion of an OMQN model, with the evolution of two extremely simple TCP connections ${ }^{3}$. White boxes are queues in $\mathcal{Q}$, solid lines represent possible transitions between states. $\mathbf{U}_{x}$ and $\mathbf{V}_{x}$ (in shaded circles) are two customers (TCP connections), $x$ being their current class. Dashed lines indicate the customer movements over the OMQN model.

$\mathbf{U}$ enters the system in class 4 (with four segments to transfer). Let us assume that $\mathbf{U}$ does not experience any loss. One segment is transmitted from $\mathrm{FE}_{1}$, and the correct reception of the first ACK after one $\overline{\mathrm{RTT}}$ triggers the transition to $\mathrm{FE}_{2}$, where two segments are transmitted. Receiving the ACK of the second segment (after another $\overline{\mathrm{RTT}}$ ), enables $\mathbf{U}$ to transmit the fourth segment in queue $\mathrm{FE}_{3}$, thus reaching class 0 , moving to $q_{M}$, and finally leaving the OMQN.

$\mathbf{V}$, instead, enters the system in class 3 (with three segments to transfer), and we assume in this case that the second segment is lost while traversing the IP network. The transmission of the first three segments proceeds as for $\mathbf{U}$; however, since the ACK of the second segment is not received, the retransmission timer of $\mathbf{V}$ expires. $\mathbf{V}$ goes to queue $\mathrm{ET}_{2}$ and retransmits the segment from queue $R_{1}$. Notice that the class

\footnotetext{
${ }^{2}$ We use here the same conventions used in [7,8] in naming queues. The number associated with the queue always indicates the current window size, while letters identify the protocol phase. For instance first exponential growth (FE) indicates the first slow start, where the slow start threshold is still undefined; exponential growth timeout (ET) indicates the wait for a timeout to expire during a slow start; $L$ a congestion avoidance phase (linear growth); $R$ the retransmission of a segment.

${ }^{3}$ For a detailed description of the OMQN model's queues and transitions, the reader is referred to [7].
} 


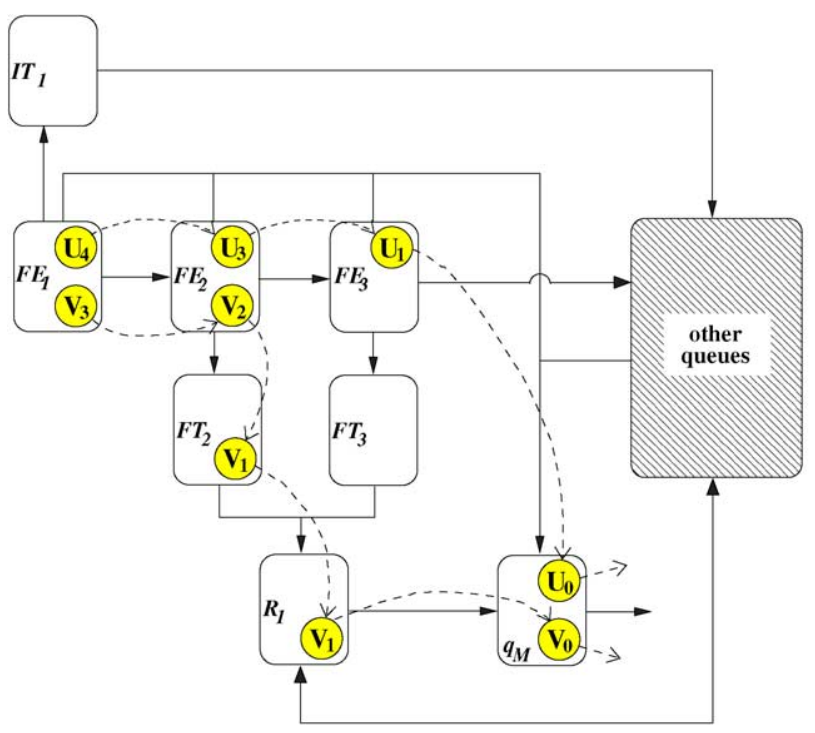

Fig. 1. Samples of connections evolution within a simplified TCP OMQN model.

is decremented by one going from $\mathrm{FE}_{2}$ to $\mathrm{ET}_{2}$, since only one of the two segments transmitted from $\mathrm{FE}_{2}$ is lost. If the segment is retransmitted successfully, a cumulative ACK (we do not consider the delayed ACK option) allows the customer class to reach 0 , so that $q_{M}$ is entered and $\mathbf{V}$ can leave the OMQN, having completed the transfer.

A complete OMQN model of TCP NewReno connections is shown in Fig. 2, under the assumption that the maximum window size is 10 segments (this is the smallest value that allows us to show a full model; larger window sizes only produce an increase of the number of queues, but no new types of queues are added). Queues in OMQN are arranged in a matrix pattern: all queues in the same row correspond to similar protocol states, and all queues in the same column correspond to equal window size. Transitions arriving to the closing queue $q_{M}$ are not shown in the figure to avoid cluttering of the graph. The OMQN models TCP dynamics precisely accounting for startup effects, timeouts due to consecutive losses and the effects of multiple segment losses. The fast recovery algorithm is also modeled. For more details, the reader is referred to [5-8].

\section{Computation of the completion time distribution}

Depending on the occurrence of losses, the life of an $N$-segment TCP connection may exhibit many different behaviors, and may correspondingly have different durations. Each behavior is represented by a possible path followed by a customer through the OMQN. Therefore, by properly exploring and analyzing the set of possible paths in the OMQN, we can compute the distribution of the completion time of an $N$-segment TCP connection.

In particular, we associate two values with each path: the probability that a customer follows the path, and the delay experienced along the path. We then combine probabilities and delays of all paths to derive the completion time distribution. 


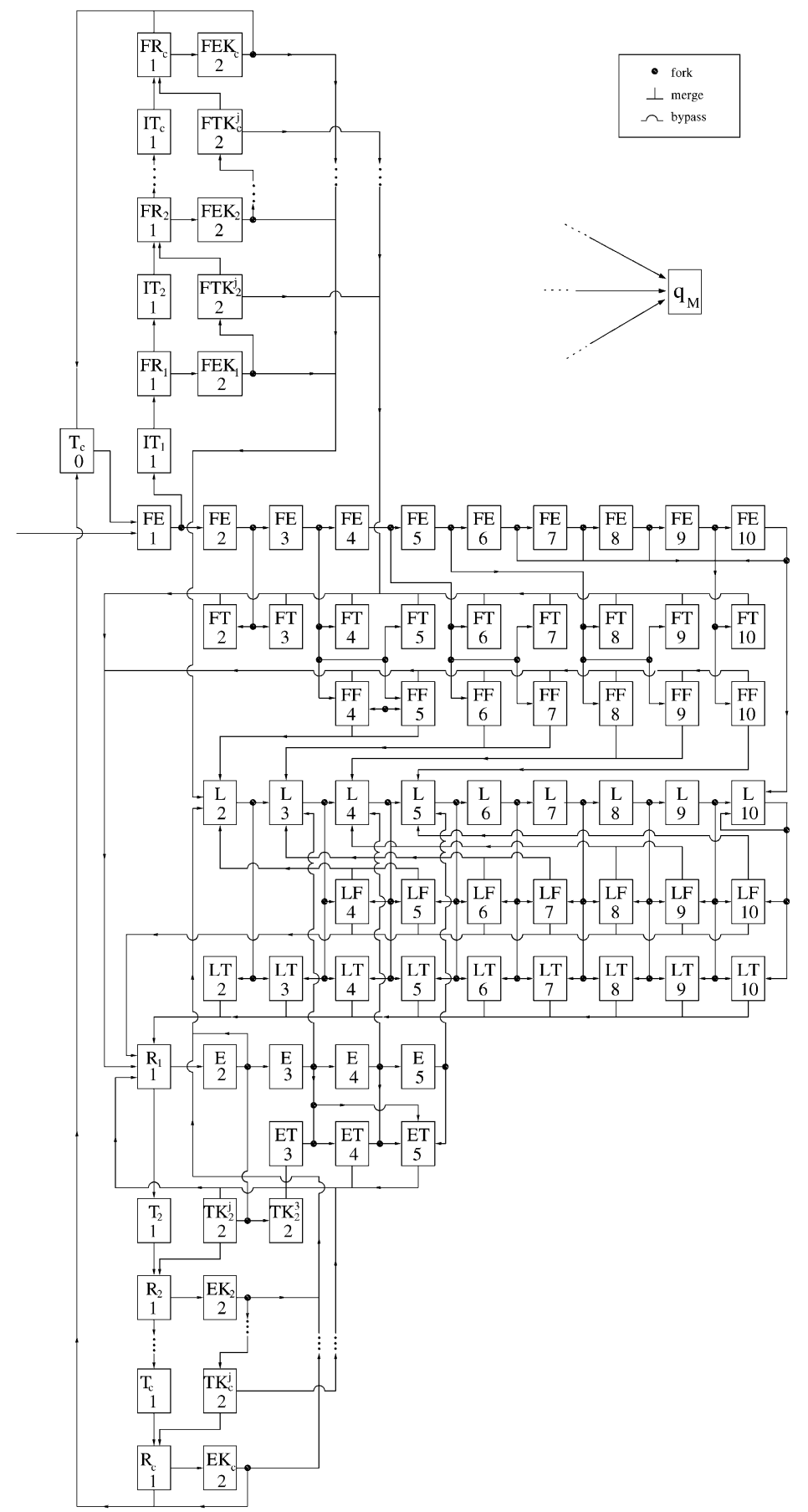

Fig. 2. The OMQN model of TCP NewReno. 
Since a path represents the dynamics of a TCP connection, it is convenient to describe it with the ordered list of states visited by the customer representing the connection. More formally, define path $i$, denoted by $h_{i}$, as the ordered list of the $n_{i}$ states visited by a connection during its lifetime:

$$
h_{i}=<\mathbf{s}_{1}, \mathbf{s}_{2}, \ldots, \mathbf{s}_{n_{i}}>
$$

where $\mathbf{s}_{k}=\left(q_{k}, c_{k}\right)$ is the $k$ th state visited by the connection. In general, different paths have different number $n_{i}$ of visited states. Denote by $\mathcal{H}$ the set of all possible paths, $\left\{h_{i}\right\}$. Due to the structure of the queuing network, the set $\mathcal{H}$, as well as the path lengths $n_{i}$ s, are finite. In fact, a customer can never visit the same queue in the same class more than once: it either will never visit the same queue again, or will visit the queue after at least one successful packet transmission, which yields a decrease in the class number. As already mentioned, we associate with path $h_{i}$ the following values:

- the probability $P\left(h_{i}\right)$ that the customer follows path $h_{i}$;

- the delay $D\left(h_{i}\right)$ incurred by the customer to follow path $h_{i}$ in the queuing network.

The probabilities $T\left(\mathbf{s}_{k}, \mathbf{s}_{k+1}\right)$ that describe the customers' transition from queue $q_{k}$ to queue $q_{k+1}$ while the customer class changes from $c_{k}$ to $c_{k+1}$ are all independent. Because of this independence, the probability $P\left(h_{i}\right)$ can be computed as follows:

$$
P\left(h_{i}\right)=T\left(\mathbf{s}_{1}, \mathbf{s}_{2}\right) T\left(\mathbf{s}_{2}, \mathbf{s}_{3}\right) \cdots T\left(\mathbf{s}_{n_{i}-1}, \mathbf{s}_{n_{i}}\right)=\prod_{k=1}^{n_{i}-1} T\left(\mathbf{s}_{k}, \mathbf{s}_{k+1}\right) .
$$

The delay associated with path $i$ is given by

$$
D\left(h_{i}\right)=\sum_{k=1}^{n_{i}} L\left(\mathbf{s}_{k}\right)
$$

where $L\left(\mathbf{s}_{k}\right)$ is the latency (or service time) of queue $q_{k} . D\left(h_{i}\right)$ represents the $N$-segment connection completion time, given that the connection behaves according to path $i$.

Since all the paths $h_{i} \in \mathcal{H}$ are mutually exclusive, the cumulative distribution function $F_{X}(t)$ of the random variable $X$ which represents the $N$-segment TCP connection completion time can be computed as

$$
F_{X}(t)=P\{X \leq t\}=\sum_{i \in \mathcal{H}_{t}} P\left(h_{i}\right) \quad \text { with } \quad \mathcal{H}_{t}=\left\{h_{i} \mid D\left(h_{i}\right) \leq t\right\} \subset \mathcal{H} .
$$

Unfortunately, since the cardinality of $\mathcal{H}$ follows an exponential law with the number $N$ of segments to be transferred over the connection, the exact computation of $F_{X}(t)$ is not feasible in most realistic cases, i.e., for connections longer than few segments. Following a deterministic approach, the authors in [13] set a maximum of 10 segments per connection.

Since the exact computation of $F_{X}(t)$ is not feasible, we are interested in developing a general methodology for the approximation of $F_{X}(t)$ reducing the complexity of the computation, while still closely approximating the distribution, both for small values of $t$, and for its tail as $t \rightarrow \infty$. Moreover, it should be possible to reduce the approximation error with an arbitrary precision.

The basic idea of the procedure is the following. We approximate the random variable $X$ with a variable $X^{\prime}$ that is distributed on a smaller number of possible values, i.e., we discretize the values that 
$X^{\prime}$ can assume. Then, instead of computing $F_{X}(t)$ as in (3), after having derived all the path probabilities and delays, we compute $F_{X^{\prime}}(t)$ while we are still generating the possible paths. In particular, while we are discovering paths, each transition from one queue to another contributes to the computation of the probability and of the delay of those paths that include the transition.

Let the random variable $Y_{\mathbf{s}}$ denote the first arrival time to state $\mathbf{s}$, that is, the time spent by the TCP connection from set-up until state $\mathbf{s}$ is entered. ${ }^{4} Y_{\mathbf{s}}$ is a discrete random variable that can assume values in $\Re$, exactly like $X$. We can write the recursive expression for its probability mass function:

$$
P\left\{Y_{\mathbf{s}}=t\right\}=\sum_{\mathbf{r} \in \mathcal{S}} P\left\{Y_{\mathbf{r}}=t-L\left(q_{r}\right)\right\} T(\mathbf{r}, \mathbf{s}),
$$

where the transition probability $T(\mathbf{r}, \mathbf{s})$ from state $\mathbf{r}$ to state $\mathbf{s}$ is equal to zero if the transition is not possible. If from state $\mathbf{r}$ a transition to $\mathbf{s}$ occurs with probability $T(\mathbf{r}, \mathbf{s})$, we deduce that with this probability the delay is equal to the delay to reach $\mathbf{r}$ plus the time spent in queue $q_{r}$, i.e., $L\left(q_{r}\right)$. We accordingly update the distribution of $Y_{\mathbf{s}}$. By so doing, the distribution of $Y_{\mathbf{s}_{0}}$, where $\mathbf{s}_{0}=\left(q_{M}, 0\right)$ is the end state of all paths, coincides with the distribution of $X$.

The starting point of the recursive procedure is the definition of the distribution for state $\mathbf{s}=\left(\mathrm{FE}_{1}, N\right)$, which is deterministic with 0 delay $\left(P\left\{Y_{\mathrm{FE}_{1}, N}=0\right\}=1\right)$, since the entry point into the queueing network is always queue $\mathrm{FE}_{1}$ in class $N$.

It is worth noticing at this point, that if $X$ and $Y_{\mathbf{s}}$ assume values in $\Re$, the complexity of the computation of $F_{X}(t)$ and $F_{Y_{\mathrm{s}}}(t)$ is equivalent. Discretizing the support of the random variable, instead, leads to the merging of large numbers of paths, which decreases the complexity of the computation of $F_{Y_{\mathrm{s}_{0}}}(t)$ with the recursive approach. The dynamics of TCP, which evolves in multiples of the round trip time, and the structure of the OMQN model, with a low connectivity degree, also help the process, since paths tends to cluster together around integer multiples of the round trip time.

Let us now focus on the discretization of $Y_{\mathbf{s}}$, which is a critical aspect of the recursive procedure in (4). Rather than discretizing the support of $Y_{\mathrm{s}}$ a priori, we chose to dynamically adjust the set of discrete values of $Y_{\mathrm{s}}$ during the recursion. Suppose that, as shown in the upper part of Fig. 3, at a given step of the recursion the probability mass function (pmf) of $Y_{\mathrm{s}}$ is represented by a set of values $t_{i}$, with probability $P_{i}$. Suppose also that the transition from $\mathbf{r}$ to $\mathbf{S}$ adds a new element to the pmf of $Y_{\mathbf{s}}$, which corresponds to abscissa $t^{*}$ with probability $P^{*}=P\left\{Y_{\mathbf{r}}=t^{*}-L\left(q_{r}\right)\right\} T(\mathbf{r}, \mathbf{s})$. As shown in the lower part of Fig. 3, we have two possibilities:

1. introduce a new abscissa $t^{*}$ in the pmf, with probability $P^{*}$ (lower left part of the figure);

2. look for the abscissa $t_{k}$ closest to $t^{*}$, and update the pmf by moving $t_{k}$ to $\left(P_{k} t_{k}+P^{*} t^{*}\right) /\left(P_{k}+P^{*}\right)$, associating with the new abscissa a probability $P_{k}+P^{*}$.

The choice between the introduction of a new abscissa in the discretization of $Y_{\mathbf{s}}$ (case 1) or the simple update of the pmf (case 2), is based on the ratio between $t_{k}$ and $t^{*}$. A new abscissa is introduced if

$$
\left|\frac{t_{k}-t^{*}}{t^{*}}\right|>R
$$

\footnotetext{
${ }^{4}$ As it was previously noted, a customer can never visit the same queue again in the same class, so the first arrival to a state is also the last one. Not all paths reach a given state; for these paths, the first arrival time can be defined as being a large finite arbitrary value.
} 

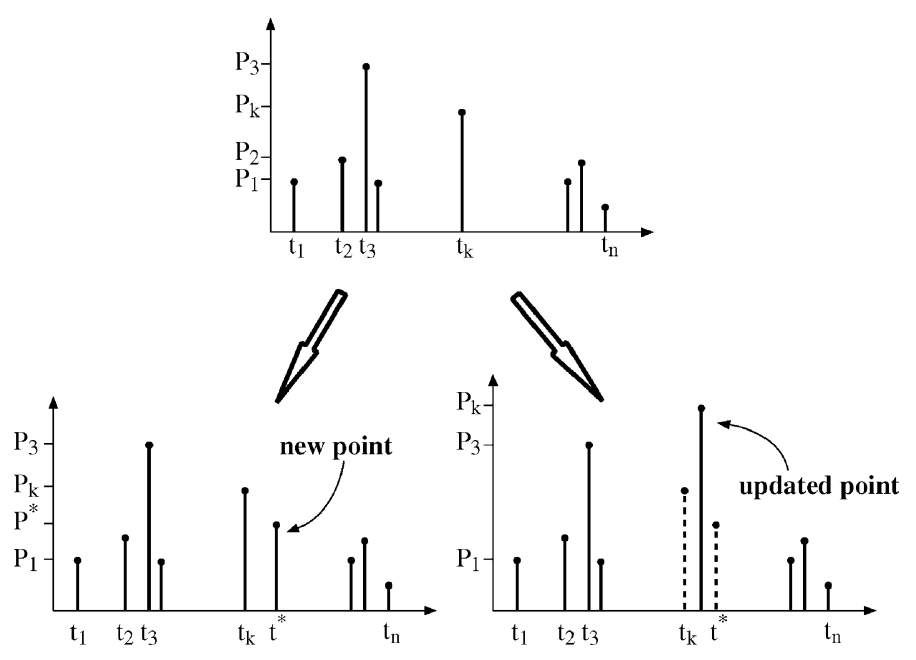

Fig. 3. Sketch of the recursive procedure to derive the probability mass function of $Y_{\mathrm{s}}$.

By basing the decision on the above ratio criterion, abscissas are allowed to range over different time scales, and tend to be equally spaced in a logarithmic scale. This is an interesting feature of our approach, since we are interested in accurately estimating the tail of the TCP connection completion time distribution. Notice also that the described discretization procedure also nicely fits the distribution shape (which is typically monotonically decreasing), making the discretization less dense where the probability mass is small. Clearly, the smaller the parameter $R$, the more accurate the discretization of $X$. As $R \rightarrow 0$ the approximating distribution converges to $F_{X}(t)$, thus ensuring that the error can be made as small as desired.

The choice of $R$ is easy, since it represents, approximately, the inverse of the number of approximating points that are used for each decade of the abscissa. The impact of $R$ on the method's precision can be observed in Fig. 4, which shows the cumulative distribution function (CDF) of the completion time of 100 -segment TCP connections in a single-bottleneck network under load 0.85 . With $R=0.1$ we can
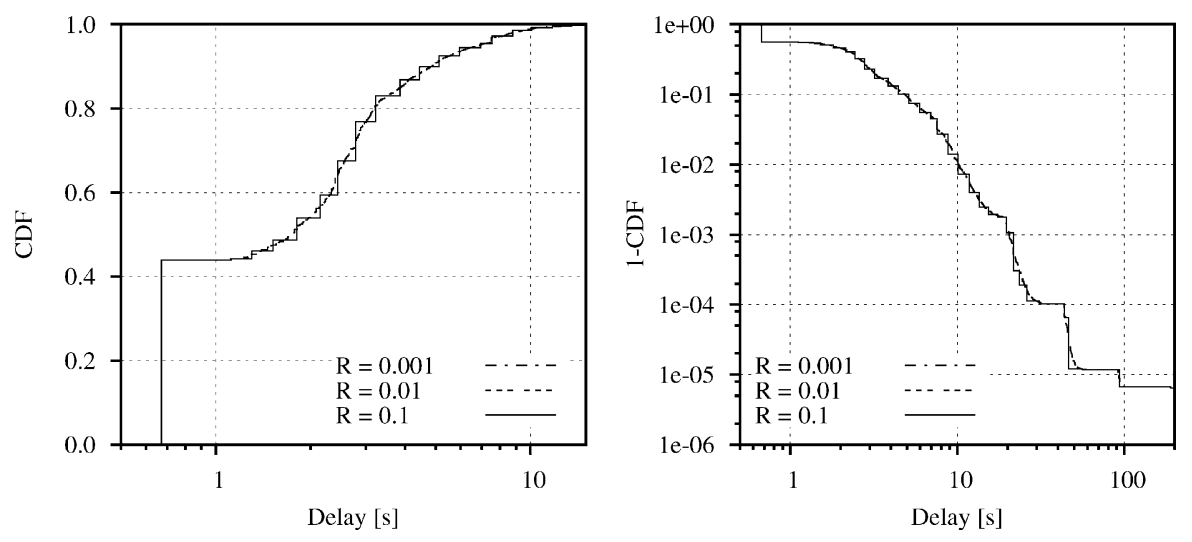

Fig. 4. Impact of $R$ on the distribution of the TCP connection completion time. 
observe a stepwise behavior in the CDF, due to a too coarse discretization, while with both $R=0.01$ and $R=0.001$ the CDF becomes smooth, and the discretization is acceptable. The same effect of $R$ can be seen on the tail of the distribution on the right hand plot of the figure, which shows the complementary CDF in log-log scale.

As similar behavior was observed also in more complex scenarios; therefore, we selected the value $R=0.01$ for the derivation of numerical results that will be discussed in Section 5 .

The speed of the algorithm can be further improved by dropping some of the abscissas from the distribution of $Y_{\mathbf{s}}$. Instead of always introducing a new abscissa in the first case, we choose to introduce it only if the threshold $P^{*}>P_{\min }$ is met. Since $P^{*}$ aggregates the probability of all the paths reaching state $\mathbf{r}$ with a delay near $t^{*}-L\left(q_{r}\right)$, the cut-off criterion ensures that no significant point can be dropped, because it is a sum of a large number of small probability paths.

For the derivation of numerical results we selected the value $P_{\min }=10^{-15}$ which resulted in negligible errors, namely the total probability of all the dropped paths was always less then $10^{-10}$.

Summarizing, we can say that the proposed method is a mixture of three techniques: discretization of the time scale, aggregation of paths reaching a state in nearly the same time, and dropping irrelevant paths from the calculation. We stress once more that the computational complexity is small and allows the exploration of realistic cases. Comparing the numerical approach to the $n s-2$ simulations we used for validation, the difference in CPU time ranges between three and four orders of magnitude.

\section{Validation and results}

The objective of this section is twofold. First, we validate our model by comparison against simulation results, thus proving that the model is able to provide extremely accurate results. Second, by using only the analytical approach and taking advantage of its computational efficiency, we extensively study the QoS perceived by TCP users in terms of completion time quantiles. We therefore show that the proposed analytical method can be very helpful in the context of QoS-aware planning of IP networks, allowing the determination of the system parameters that yield a specified maximum completion time to a given percentage of TCP connections of any given length.

\subsection{Model validation}

In order to validate the technique, we compare its results against simulation experiments under various network scenarios. As a simulation tool we employ the $n s-2$ simulator [19], which has become the standard for the validation of analytical models of TCP. Throughout this section we use the NewReno version of TCP, although the method can be applied to other versions of the protocol as well.

Evaluating confidence intervals for distributions is not a trivial task. In order to obtain an indication of the results reliability, we build an indication function:

$$
I_{X_{i}<x}= \begin{cases}1, & \text { if } X_{i}<x, \\ 0, & \text { otherwise }\end{cases}
$$

where $x$ is the (discretized) time variable and $X_{i}$ is the random variable describing the completion time of the $i$ th connection. 


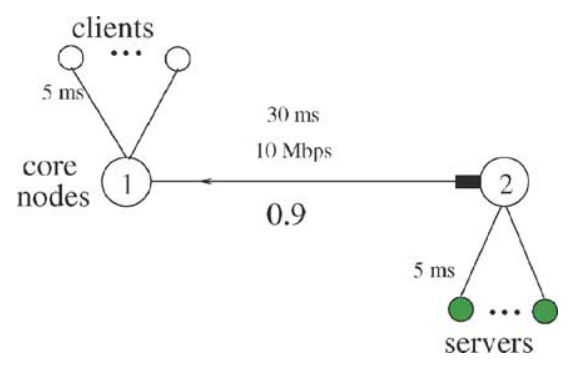

Fig. 5. Single bottlenecks scenario with homogeneous connections.

For each value of $x$ the average of these indicators:

$$
F_{n}^{*}(x)=\frac{1}{n} \sum_{i=1}^{n} I_{X_{i}<x}
$$

can be used to estimate the confidence interval invoking the Central Limit Theorem, since the indicators are i.i.d. by construction. By so doing, confidence intervals of the whole distribution can be calculated. Results referring to simulations reports three separate curves with the same graphic tract: the middle one is the average value, while the other two (thinner lines) define the confidence interval with a confidence level of $99 \%$.

\subsubsection{Single bottleneck scenario}

We first consider a simple network scenario consisting of a single bottleneck link with capacity $C=$ $10 \mathrm{Mbps}$. The one-way propagation delay is $40 \mathrm{~ms}$. A gentle RED queue management scheme is adopted, with low and high thresholds equal to 10 and 80 segments, respectively, with loss probability at the high threshold equal to $5 \%$. The buffer size is 512 segments. The network is loaded by TCP connections, all of which transfer $N_{s}=100$ segments, the segment size is constant and equal to $S_{p}=1000$ bytes. TCP connections are generated according to a Poisson process, whose rate $\lambda$ is such that the nominal link load $\rho_{n}$ is equal to 0.9 . TCP maximum window size is 64 segments, the tic is $0.5 \mathrm{~s}$ and the initial timeout is set to $6 \mathrm{~s}$. The nominal load is defined as

$$
\rho_{n}=\frac{\lambda N_{s} 8 S_{p}}{C}
$$

where $C$ is the link capacity in Mbit/s. Computing the actual link load, including retransmissions, can be done only a posteriori, knowing the packet drop rate. The scenario is sketched in Fig. 5.

The cumulative distribution function (CDF) of the TCP connection completion time is shown in Fig. 6. The solid line reports simulation results, the two dashed lines refer to the analytical approach and differ in the way the round-trip time and segment loss probability are computed.

For the results shown in long-dashed style ("using NW model"), the analytical model of the IP network is used to derive the average round-trip time and segment loss probability; ${ }^{5}$ for the short-dashed line

\footnotetext{
${ }^{5}$ The iterative algorithm presented in [7] was used to calculate these values. This way, the use of the OMQN model is twofold: the steady-state solution is used in the iterative process to obtain averages, then its dynamic properties are used to determine the distribution.
} 

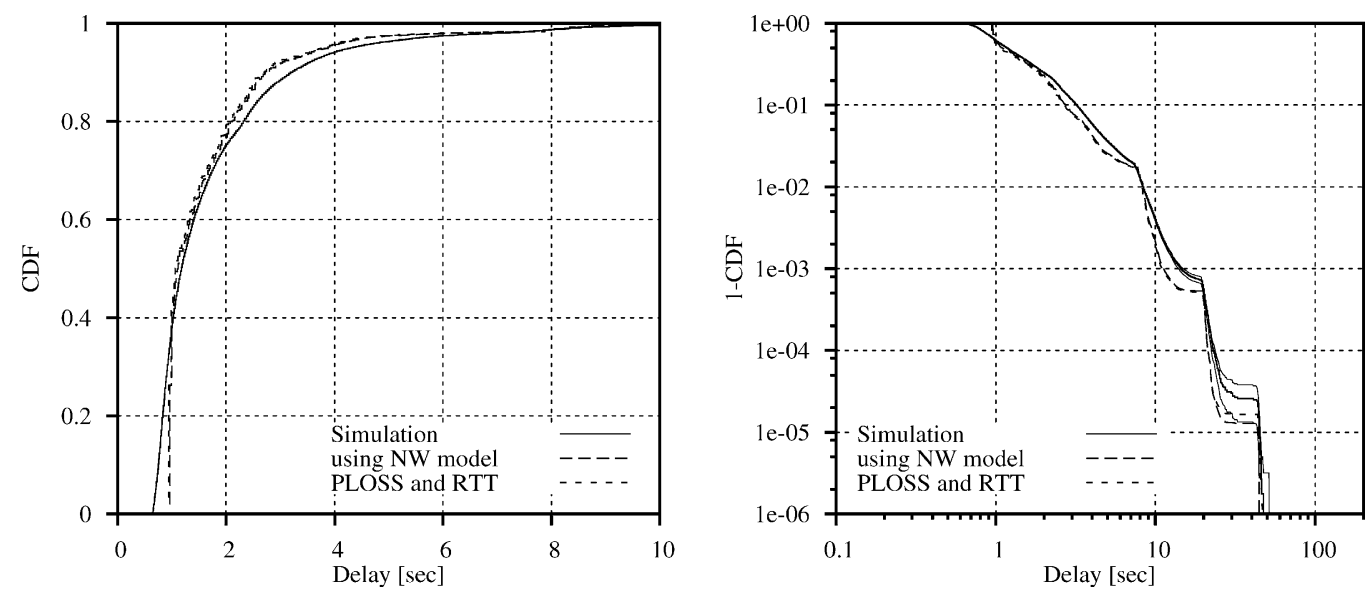

Fig. 6. CDF and CCDF of the completion time. Single bottleneck, homogeneous connections with $N_{s}=100$ and $\rho_{n}=0.9$; Analysis $\left(\overline{\mathrm{RTT}}=134 \mathrm{~ms}\right.$ and $\left.P_{L}=1.64 \%\right)$ and simulation $\left(\overline{\mathrm{RTT}}=132 \mathrm{~ms}\right.$ and $\left.P_{L}=1.61 \%\right)$.

("PLOSS and RTT"), instead, the estimates of the round-trip time and loss probability are computed by simulation. ${ }^{6}$ Showing both of these curves has two reasons:

- It allows the separation of the inaccuracy due to the IP network model from the inaccuracy in the calculation of the completion time distribution.

- It demonstrates two different uses of the completion time distribution calculation method. In the first case a network whose topology, equipment and traffic is known could be evaluated from the point of view of TCP quality with an all-analytic approach. In the second case, on the other hand, the environment in which TCP works is not important, it can be seen as a black box, where only the loss probability and the round trip time is known from some source.

The behavior of the tail of the distribution can be observed on the right hand part of Fig. 6, which shows the complement of the CDF (CCDF) in logarithmic scale. The peculiar behavior of the curve, with points where the slope changes abruptly, is due to TCP initial timeouts. The first peak around $7 \mathrm{~s}$ corresponds to the following case: after the first segment is lost, the $6 \mathrm{~s}$ initial timeout expires and the retransmission of the initial segment is successful. The second peak is around $19 \mathrm{~s}$. In this case, the first segment transmission fails for two consecutive times and the backoff mechanism applies. Despite the low computational cost, the analytical approach is able not only to catch the shape of the distribution, but also to provide extremely accurate results even for small values of the probability. On the contrary, simulations require a considerable amount of computation time, especially when, as in most of the cases of interest, low probabilities are considered.

The probability density function (PDF) of the TCP connection completion time is shown in Fig. 7. Again, while small probability parts of the PDF, of the order of $10^{-5}$ or smaller, are quite costly to obtain by simulation, the analytical approach provides reliable estimates even for extremely low values with no

\footnotetext{
${ }^{6}$ We use the same simulation as for the validation curve, although a much shorter simulation would be enough to determine these average values.
} 


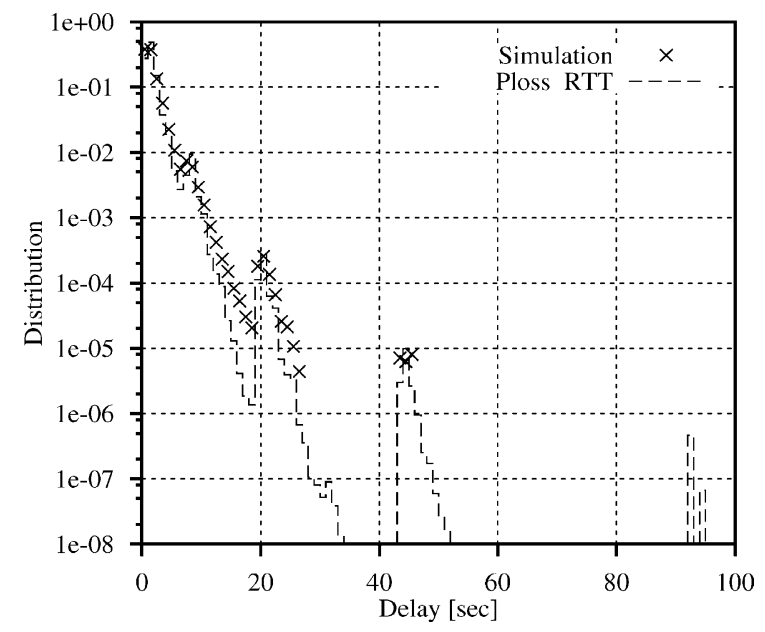

Fig. 7. Analysis and simulation. Probability density function. Single bottleneck, homogeneous connections with $N_{s}=100$ and $\rho_{n}=0.9$.

additional computational cost. We simulated 1.125 millions flows in order to obtain the curve in Fig. 7 (we plot a point if we have at least five samples), and it took $3 \mathrm{~h}$. The analytical results were computed in less than $4 \mathrm{~s}$.

We now consider shorter TCP connections that transfer only 10 segments, sharing the same singlebottleneck network described before. The CCDF is in this case shown in Fig. 8. Again, analytical estimates are very accurate. However, the curve reporting simulation results exhibits a smoother behavior. This is due to the deterministic round-trip time assumption introduced in the model. Consider for example the steep decrease of the curve when the delay is about $450 \mathrm{~ms}$ : it corresponds to the case in

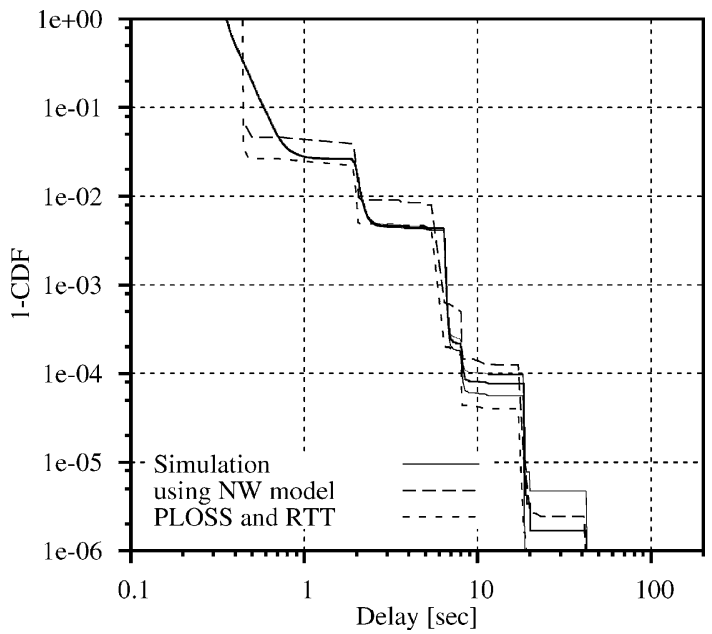

Fig. 8. CCDF, single bottleneck, connections with $N_{s}=10$ and $\rho_{n}=0.95$. Analysis $\left(\overline{\mathrm{RTT}}=117 \mathrm{~ms}\right.$ and $\left.P_{L}=0.6 \%\right)$ and simulation $\left(\overline{\mathrm{RTT}}=109 \mathrm{~ms}\right.$ and $\left.P_{L}=0.5 \%\right)$. 
which no segment losses occur. While by simulation the no loss case translates into values of completion time varying between 350 and $800 \mathrm{~ms}$ due to the variability of the round-trip time, in the analytical model only one path corresponds to the no loss case, so that a deterministic completion time is predicted.

By comparing this plot to the one in Fig. 6, which refers to 100 segment connections, it can be observed that behaviors are similar, but shorter connections lead to steeper changes in the CDF. In fact, for long connections, a large set of possible paths combine to form the CDF and this makes the curve behavior smoother.

\subsubsection{Multiple bottleneck scenario}

In order to validate our approach in a more complex network environment, we consider the three routers scenario sketched on the left hand part of Fig. 9. The traffic used is also more realistic, it consists of nonhomogeneous TCP connections: $50 \%$ of the generated connections contain 10 segments, $40 \%$ contain 20 segments, and the remaining $10 \%$ of the connections are 100 segments long. Of course, connections crossing different paths perceive different round-trip time and loss probability. The right hand side of Fig. 9 shows, by simulation only, the reason why we are interested in the completion time distribution of different TCP flows in various network conditions: differences in network conditions induce significant differences in completion time distributions. The plot shows flows traversing routers 2 and 1 (labeled "Flow $2 \rightarrow 1$ " in the figure) and flows traversing the three routers (labeled "Flow $3 \rightarrow 1$ " in the figure). Due to larger loss probability and round-trip time, the completion time for connections crossing the three routers tends to be significantly longer than the completion time of the other flows. The difference caused by the amount of data to be transferred is smaller than the difference caused by the path, specially when the distribution tail is considered (only connections of lengths 10 and 100 segments are shown for clarity).

The validation of the analytical approach in this more complex scenario is shown in Fig. 10. These results confirm the accuracy and flexibility of the model. Simulation based analysis is limited due to required time, thus the accuracy of the analytical model opens new possibilities, discussed in Section 5.2.
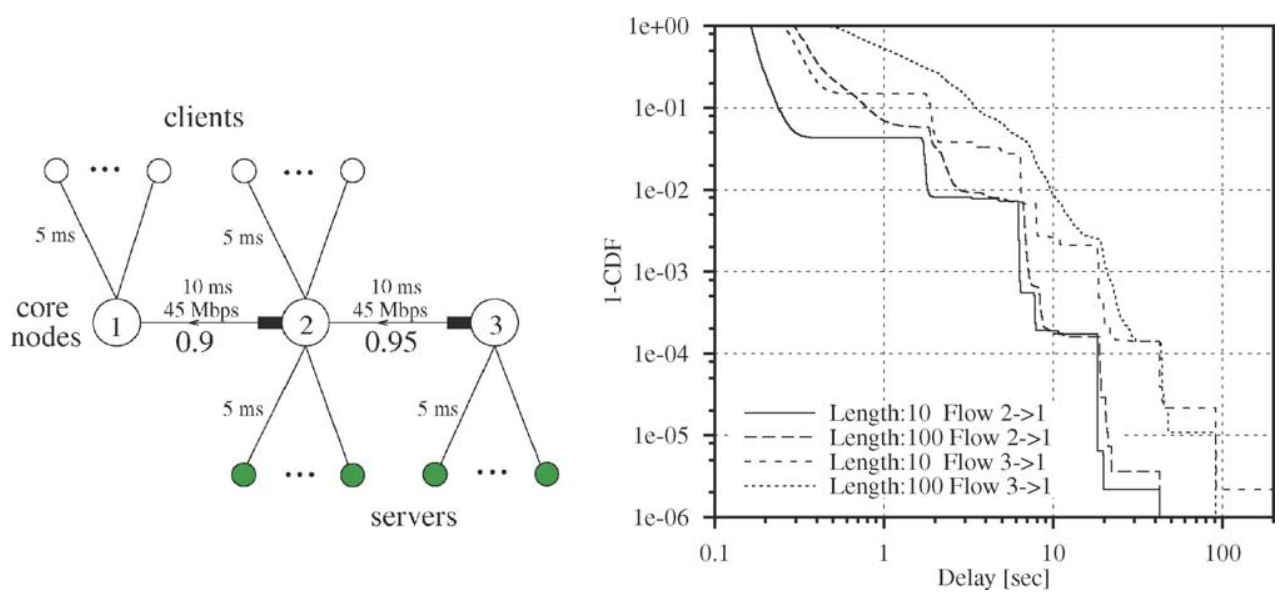

Fig. 9. Two bottlenecks scenario, non-homogeneous connections. Setup (left) and simulation results (right). Flows from router 2 to router $1\left(\overline{\mathrm{RTT}}=47 \mathrm{~ms}\right.$ and $\left.P_{L}=0.7 \%\right)$ and from router 3 to router $1\left(\overline{\mathrm{RTT}}=95 \mathrm{~ms}\right.$ and $\left.P_{L}=2.7 \%\right)$. 

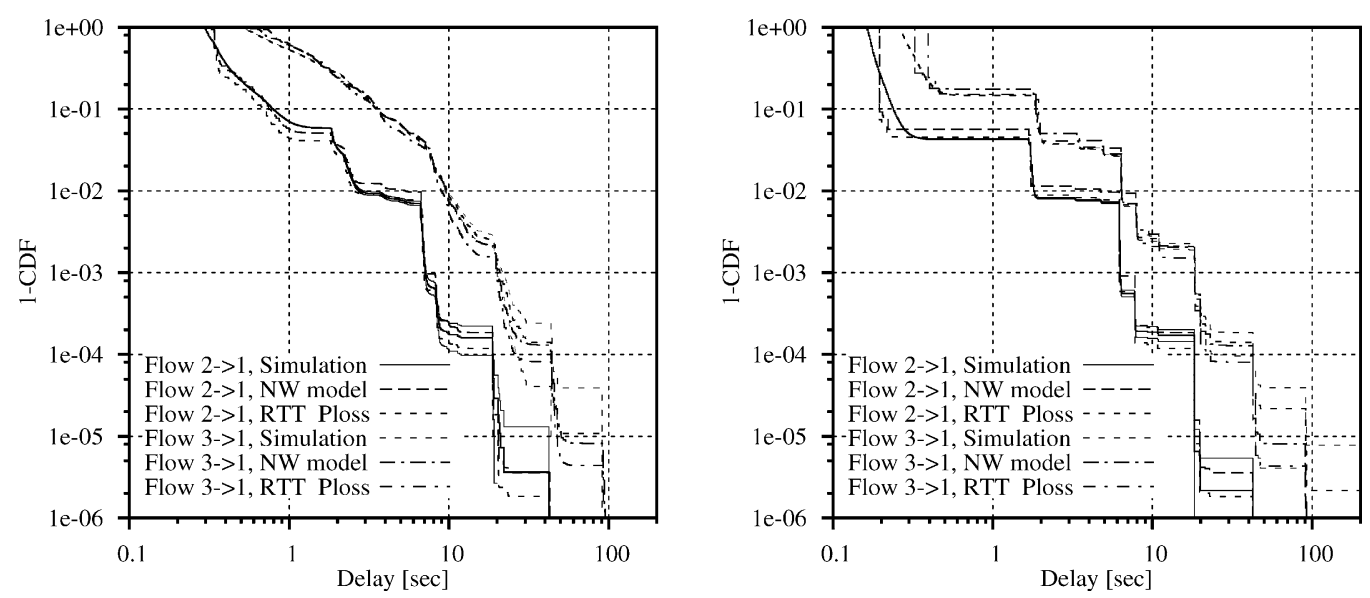

Fig. 10. Analysis and simulation. Complement of the cumulative distribution function. Two bottlenecks scenario, nonhomogeneous connections. Connection length $N_{s}=100$ (left) and $N_{s}=10$ (right).

\subsubsection{Mixed UDP and TCP traffic}

As the last part of the validation, to demonstrate the model's ability to handle a wide range of loss probabilities and even more realistic network traffic, we show a single bottleneck network overloaded with TCP and UDP traffic. In this scenario $2 \mathrm{Mbps}$ UDP traffic is generated by CBR sources, while the nominal link load of TCP connections is set to 0.85 . This overload situation resulted in a very high drop rate of $29 \%$. Fig. 11 clearly shows that the analytical model could handle even this large drop rate with good precision (the analytical model of the IP network we used for the fixed point algorithm does not support UDP traffic, therefore the curve "using NW model" is not shown).
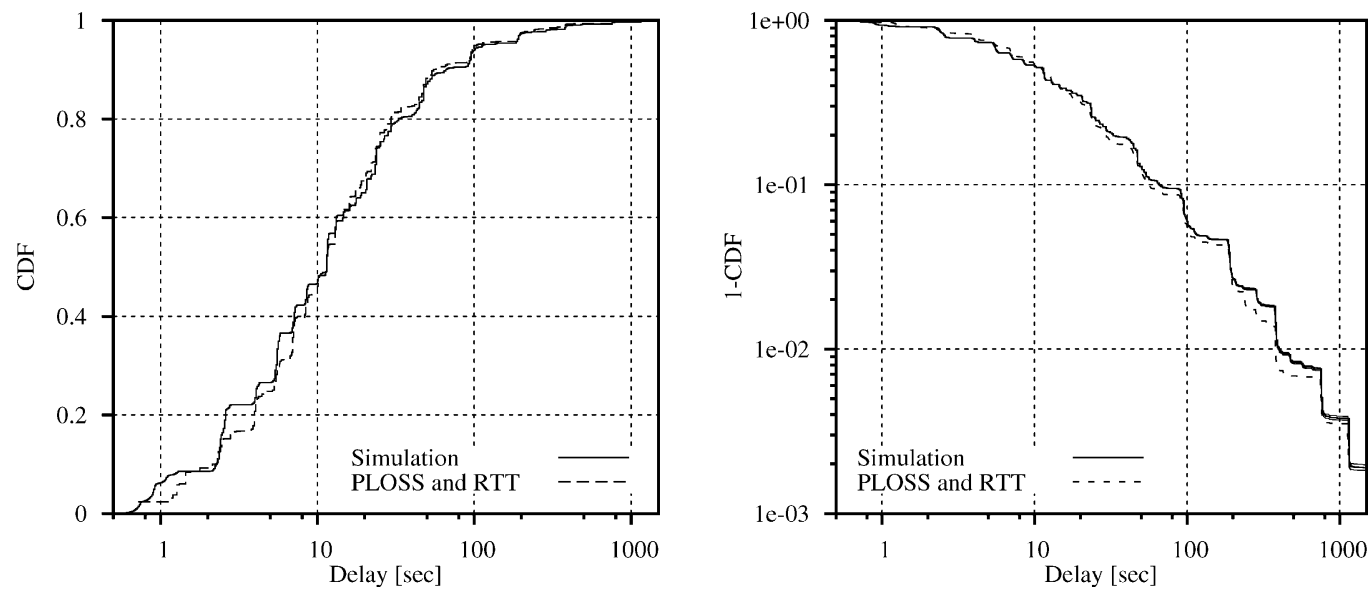

Fig. 11. CDF and CCDF. Single bottleneck scenario, mixed UDP and TCP traffic. Connection length $N_{s}=10$. Analysis and simulation $\left(\overline{\mathrm{RTT}}=175 \mathrm{~ms}\right.$ and $\left.P_{L}=29 \%\right)$. 


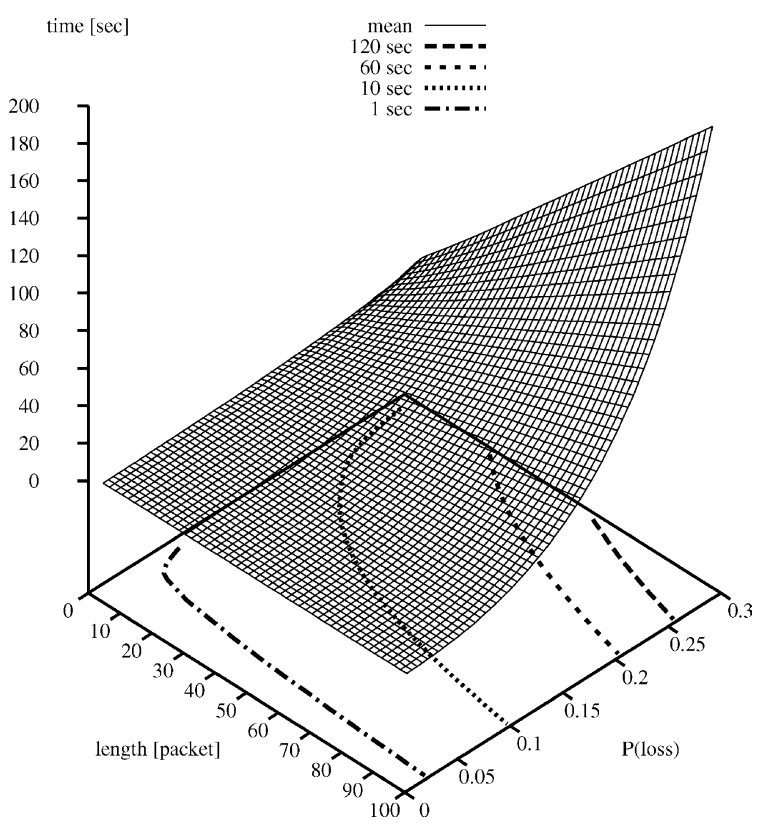

Fig. 12. Analytical results. Mean of the completion time as a function of the connection length and of the segment loss probability.

\subsection{Further results}

Besides allowing a deep understanding of the protocol dynamics, the analytical model has two major advantages over simulation. First, it is much more efficient; second, the computational cost is almost insensitive to the network scenario, the values of the network parameters, and the order of magnitude of the probability of interest. Therefore, the analytical model can be effectively used in the context of planning and performance evaluation of IP networks.

Fig. 12 reports a 3-D plot of the mean of TCP connections completion time as a function of the connection length and the segment loss probability, given a $50 \mathrm{~ms}$ round-trip time. Of course, large values of loss probability and connection length correspond to large values of the mean completion time. As known from measures and empirical models, the average completion time grows linearly with the connection length, while it grows with an hyperbolic law with the segment loss $P_{L}$.

Fig. 13 shows 0.95 and 0.99 quantiles in the same situation. It is interesting noticing that curves describing delay quantiles are not "scaled versions", of the average delay, but tend to have different shapes, with a step-wise behavior that is more pronounced as the quantile approaches the distribution tail.

Curves of the TCP connections quantiles are particularly meaningful in the context of network planning. Suppose that we need to plan a network which guarantees with confidence 0.95 to all TCP connections shorter than 100 segments, i.e., for transfers of files smaller than 100 kbytes, a completion time shorter than $10 \mathrm{~s}$. From the contour lines we learn that we need to plan a network which guarantees segment loss probability smaller than $5 \%$. A QoS target of $10 \mathrm{~s}$ with confidence 0.99 , instead, translates for the same TCP connections into the need to provide segment loss probability smaller than $3 \%$ (see the projection countour lines in Fig. 13). 

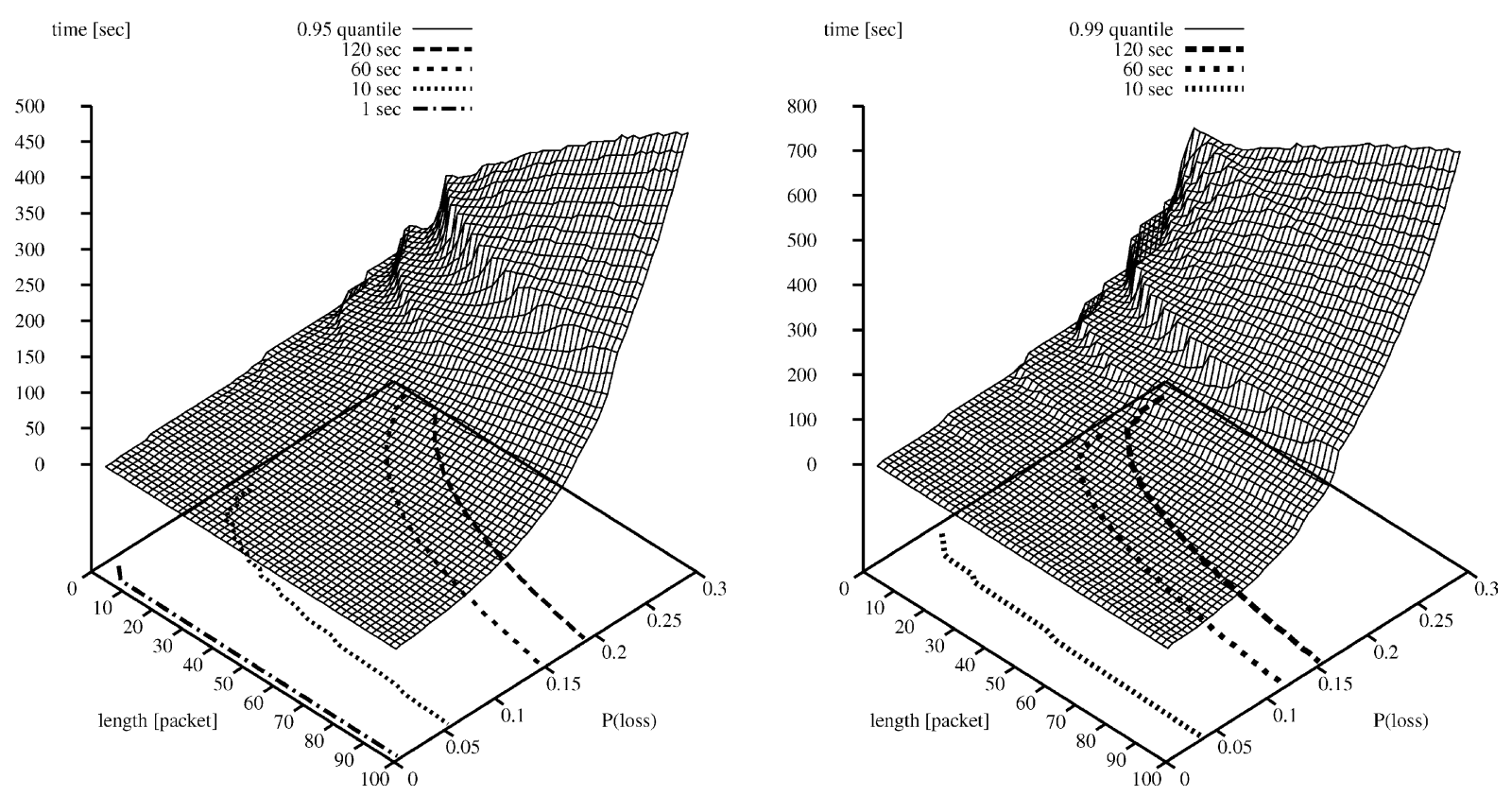

Fig. 13. Analytical results: 0.95 (left) and 0.99 (right) completion time quantiles as a function of the connection length $N_{s}$ and the segment loss probability $P_{L}$.

\section{Conclusions}

This paper has proposed and discussed a path analysis technique applied to open multiclass queuing network models of the TCP protocol that can be used to obtain reliable and accurate estimates of the distributions and quantiles of the completion time of short-lived TCP connections.

The OMQN models of TCP require as input only the packet loss probability, and the average round trip time for the TCP connections being considered. These can be either obtained through measurements and simulations, or estimated with an analytical model of the underlying IP network, that works in conjunction with the OMQN model of TCP. In all cases, the same path analysis post-processing can be applied.

Numerical results prove the flexibility and the accuracy of the proposed methodology, whose computational complexity is small and does not depend on network load or topology. Several interesting features of the completion time distribution, that cannot be simulated due to prohibitive simulation times, can be highlighted with the proposed approach.

The ability to accurately estimate quantiles of TCP connection completion times provides a very important support to the design and planning of IP networks with QoS support, and in particular can be instrumental for the specification of the service level agreements that a network operator can reliably offer to different classes of end users.

\section{Acknoledgements}

This work was supported by the Italian Ministry of Education, University and Research through the project PlanetIP and by the Hungarian Scientific Research Fund grant OTKA T30685. 


\section{References}

[1] E. Altman, K. Avrachenkov, C. Barakat, A stochastic model of TCP/IP with stationary random losses, in: ACM SIGCOMM 2000, Stockholm, Sweden, 2000, pp. 231-242.

[2] P. Barford, M. Crovella, Critical path analysis of TCP transactions, IEEE/ACM Trans. Networking 9 (3) (2001) $238-248$.

[3] A.W. Berger, Y. Kogan, Dimensioning bandwidth for elastic traffic in high speed data networks, IEEE/ACM Trans. Networking 8 (5) (2000) 643-654.

[4] N. Cardwell, S. Savage, T. Anderson, Modeling TCP latency, in: Proceedings of the Infocom 2000, Tel Aviv, Israel, 2000, pp. 1742-1751.

[5] M. Garetto, R. Lo Cigno, M. Meo, M. Ajmone Marsan, Closed queuing network models of interacting long-lived TCP flows, IEEE/ACM Trans. Networking 12 (2) (2004) 300-313.

[6] M. Garetto, R. Lo Cigno, M. Meo, M. Ajmone Marsan, A detailed and accurate closed queueing network model of many interacting TCP flows, in: Proceedings of IEEE Infocom 2001, Anchorage, AK, 2001, pp. 1706-1715.

[7] M. Garetto, R. Lo Cigno, M. Meo, E. Alessio, M. Ajmone Marsan, Modeling short-lived TCP connections with open multiclass queueing networks, Comput. Networks 44 (2) (2004) 153-176.

[8] M. Garetto, R. Lo Cigno, M. Meo, M. Ajmone Marsan, On the use of queueing network models to predict the performance of TCP connections, in: Proceedings of the IWDC 2001, Taormina, Italy, 2001, pp. 536-555.

[9] M. Garetto, M. Ajmone Marsan, R. Lo Cigno, M. Meo, On the convergence of fixed point approximations to study congested networks loaded by TCP traffic, in: Proceedings of IEEE Globecom 2003, San Francisco, CA, December 2003, pp. 3133-3137.

[10] S.H. Low, F. Paganini, J. Wang, S. Adlakha, J.C. Doyle, Dynamics of TCP/RED and a scalable control, in: Proceedings of IEEE Infocom 2002, New York, June 2002, pp. 239-248.

[11] F. Paganini, Z. Wang, S.H. Low, J. Doyle, A new TCP/AQM for stable operation in fast networks, in: Proceedings of IEEE Infocom 2003, vol. 1, San Francisco, CA, April 2003, pp. 96-105.

[12] M. Mathis, J. Semke, J. Mahdavi, T. Ott, The macroscopic behavior of the TCP congestion avoidance algorithm, Comput. Commun. Rev. 27 (3) (1997) 67-82.

[13] M. Mellia, I. Stoica, H. Zhang, TCP model for short lived flows, IEEE Commun. Lett. 2 (6) (2002) 85-88.

[14] V. Mishra, W.B. Gong, D. Towsley, Fluid-based analysis of a network of AQM routers supporting TCP flows with and application to RED, in: Proceedings of ACM SIGCOMM 2000, Stockholm, Sweden, 2000, pp. 151-160.

[15] M. Mitzenmacher, R. Rajaraman, Towards more complete models of TCP latency and throughput, J. Supercomput. 20 (2) (2001) 137-160.

[16] J. Padhye, V. Firoiu, D. Towsley, J. Kurose, Modeling TCP reno performance: a simple model and its empirical validation, IEEE/ACM Trans. Networking 8 (1) (2000) 133-145.

[17] B. Sikdar, S. Kalyanaraman, K.S. Vastola, An integrated model for the latency and steady-state throughput of TCP connections, Perform. Eval. 46 (2-3) (2001) 139-154.

[18] B. Sikdar, S. Kalyanaraman, K.S. Vastola, Analytic models and comparative study of the latency and steady-state throughput of TCP Tahoe, Reno and SACK, in: Proceedings of IEEE GLOBECOM, San Antonio, TX, November 2001, pp. $1781-1787$.

[19] ns-2, network simulator (ver.2), Lawrence Berkeley Laboratory, URL: http://www.mash.cs.berkeley.edu/ns. 